\title{
Spectrally resolved measurements of absorption of broadband UV light-emitting diodes by combustion radicals
}

\author{
Logan W. White* and Mirko Gamba ${ }^{\dagger}$ \\ University of Michigan, Ann Arbor, MI, 48109
}

\begin{abstract}
A novel absorption diagnostic is proposed which probes combustion radicals in flames using broadband UV excitation produced by light-emitting diodes and measures the spectrum of the light transmitted through the absorption path. Fitting of the measured spectra using a model of the absorption allows values for temperature, species concentration, and potentially other properties to be inferred. This work provides an outline of how these types of measurements may be made in experiments and how the results can be analyzed and interpreted. The diagnostic is demonstrated by inferred measurements of temperature and hydroxyl radical concentration in a laminar methane-air flame.
\end{abstract}

\section{Nomenclature}

$\sigma_{a b s}$ Absorption cross-section, $\mathrm{cm}^{2}$

$T$ Temperature

$P \quad$ Pressure

$\tilde{\nu} \quad$ Wavenumber, $\mathrm{cm}^{-1}$

A Einstein A coefficient, $\mathrm{s}^{-1}$

$c \quad$ Speed of light, $\mathrm{cm} / \mathrm{s}$

$\tilde{\nu}_{0} \quad$ Central wavenumber of a particular spectral line, $\mathrm{cm}^{-1}$

$J \quad$ Total angular momentum quantum number

$f_{b} \quad$ Boltzmann population fraction of the lower state of a particular transition

$\phi \quad$ Spectral absorption lineshape for a particular transition, $\mathrm{cm}$

$\eta \quad$ Optical transmission

I Transmitted optical intensity, $\mathrm{W} / \mathrm{cm}^{2} / \mathrm{cm}^{-1}$

$I_{0} \quad$ Initial optical intensity, $\mathrm{W} / \mathrm{cm}^{2} / \mathrm{cm}^{-1}$

$n \quad$ Local number density, $\mathrm{cm}^{-3}$

$X_{O H} \mathrm{OH}$ mole fraction

$l \quad$ Absorption path length, $\mathrm{cm}$

Subscript

$i \quad$ Summation index

$m \quad$ Indicates incorporation of instrument broadening effects

Superscript

' Indicates excited electronic state

"Indicates ground electronic state

\section{Introduction}

The hydroxyl radical, $\mathrm{OH}$, is an important chemical intermediate found in reaction zones and hightemperature regions of virtually every air-breathing combustion system. Many laser-based spectroscopic

*Graduate Student, Dept. of Aerospace Engineering.

${ }^{\dagger}$ Assistant Professor, Dept. of Aerospace Engineering, AIAA Member. 
strategies have been developed to measure $\mathrm{OH}$ density ${ }^{1,2}$ and temperature ${ }^{3-6}$ in combustion environments. Quantitative measurements become difficult in high pressure systems and one of the primary reasons is the reduction in signal intensity due to spectral line broadening exceeding the bandwidth of the excitation source. ${ }^{7-11}$

Broadband excitation sources, such as newly available UV light-emitting diodes (LEDs), offer a way to mitigate the loss in signal intensity suffered at high pressure by techniques based on relatively narrow lasers. Battles and Hanson ${ }^{12}$ achieved improved signal levels by increasing the bandwidth of their laser system, showing the value of the nascent broadband nature of the spectrum emitted by commercial LEDs. Previous works in the area of plasma diagnostics have used broadband UV LEDs to measure OH densities and temperatures in RF-generated plasmas. ${ }^{13-16}$ Others have proposed using LEDs to produce the probe beam for cavity ring-down spectroscopy experiments ${ }^{17}$ and for NOx and Ozone sensors in engine exhausts. ${ }^{18-20}$ These studies demonstrate the potential to develop LED-based absorption and fluorescence sensors that can measure number densities and temperatures with relatively little sensitivity to pressure. In this work, we implement and demonstrate the approach using absorption of $\mathrm{OH}$ as an example, however, the method can be readily extended to other intermediate species of combustion.

While the measurement of radicals has been investigated by several researchers in the plasma dynamics field, the use of LEDs as the light source for absorption measurements of intermediates in combustion systems has not received as much attention. Schorsch et al. ${ }^{21}$ used spectrally integrated measurements of LED absorption to detect the presence of $\mathrm{OH}$ and methylidyne radicals, $\mathrm{CH}$, in atmospheric pressure methane-air flames. That work demonstrated the ability of LED-based absorption techniques to measure flame radicals in a combustion environment with some temporal and spatial (beam diameter of $1 \mathrm{~mm}$ to $4 \mathrm{~mm}$ ) resolution. While the results of that study demonstrated a qualitative measurement of the concentration profile of radicals in a flame, the spectrally integrated nature of the measurement prevented a quantitative decoupling of the effects of temperature and number density on $\mathrm{OH}$ absorption.

The novel approach of leveraging spectrally resolved measurements is advantageous because many properties such as concentration, pressure, and temperature effect the absorption spectrum, but they do so in subtly different ways and their influences can be separated through careful analysis. This means that in general the diagnostic can be either relatively insensitive to changes in certain properties such as pressure, or potentially able to measure those properties simultaneously with temperature and species concentration. The initial examination presented in this work is focused on isobaric flames, removing pressure as a variable from the problem. Future work will look at the use of this type of diagnostic in flames at elevated pressures to investigate the capability of this diagnostic to provide sensing of flow properties in production scale combustors.

The present work will outline an approach for making these types of experimental measurements and how to interpret the data from them. This involves the development of a model of $\mathrm{OH}$ absorption that can be used for the purpose of spectral fitting, an experimental design including characterization of a spectral measurement instrument, and a method for finding optimized fits to measured spectra in order to infer temperature and species concentration values. This work will describe the problem of spectral fitting and challenges associated with inferring information from the measured absorption spectra. It will also present a description of how resolution enhancement techniques may be used to improve spectral fitting performance in a way similar to their use in Fourier transform infared spectroscopy (FTIR). ${ }^{22,23}$ These techniques and their results are demonstrated by a series of measurements made on a laminar methane-air flame. An estimation of the uncertainty of those measurements and a discussion of how uncertainty in these types of measurements should be interpreted is given.

\section{Theory and Modeling}

In order to perform spectral fitting of the $\mathrm{OH}$ absorption measured in a flame, a model for the $\mathrm{OH}$ absorption spectrum must be developed. Spectroscopic constants for the hydroxyl molecule, including the line positions and Einstein A coefficients, were taken from data tables provided in the LIFBASE software package. ${ }^{24}$ The total spectral absorption cross-section of $\mathrm{OH}$ under given conditions, $\sigma_{a b s}(T, P, \tilde{\nu})$, may then be taken as the summation of all relevant absorption lines within the bandwidth of interest. If thermal equilibrium of the internal energy levels is assumed, such that a Boltzmann energy distribution is valid, then this total absorption cross-section takes the form given in Eq. (1) where $i$ represents all relevant transitions. 


$$
\sigma_{a b s}(T, P, \tilde{\nu})=\sum_{i} \frac{A_{i}}{8 \pi c \tilde{\nu}_{0, i}^{2}}\left(\frac{2 J_{i}^{\prime}+1}{2 J_{i}^{\prime \prime}+1}\right) f_{b, i}(T) \phi_{i}(T, P, \tilde{\nu})
$$

The lineshape of a particular transition, $\phi_{i}$, is modeled as a Voigt lineshape with the parameters determined by estimates of Doppler and collisional broadening widths. The magnitude of collisional broadening was estimated using a linear best fit in pressure of broadening data reported in nearly stoichiometric methaneair flames by Atakan et. al. ${ }^{9}$

It is important to note that Eq. (1) invokes a broad definition of temperature under the assumption of thermal equilibrium of $\mathrm{OH}$ internal energy levels. In this case, the temperatures used to determine the local gas number density, the Doppler broadening of each spectral line, and to determine the Boltzmann population fractions in the ground electronic level are all taken to have the same value. While this assumption is reasonable for low-speed, atmospheric pressure flames, it may not always be in the case and this model can be modified to account for differences between the translational, rotational, and vibrational temperatures. The spectral fitting used for the measurements in this work primarily depends on the rotational temperature that determines the relative ground state rotational populations and therefore the relative strengths of absorption spectrum lines. For this reason, the diagnostic is primarily measuring the rotational temperature of the $\mathrm{OH}$ molecules along the absorption path. Because thermal equilibrium is assumed, the term temperature and $\mathrm{OH}$ rotational temperature will be used interchangeably. In other applications were thermal equilibrium is not expected, the diagnostic can still be implemented and will be primarily sensitive to the $\mathrm{OH}$ rotational temperature with a secondary capability to estimate translational temperature through spectral line-widths.

Once the spectral absorption cross-section is computed, the Beer-Lambert law may be used to model the transmission spectrum, $\eta$, as shown in Eq. (2). Implicit in this formulation is the assumption that the conditions are constant along the absorption path length. This assumption is believed to be reasonable because of the design of the burner to produce a "flat flame" with radially uniform conditions, which has been verified with measurements of the radial temperature profile above our burner. The effect of instrument broadening, which is discussed in the next section, must be accounted for in the absorption spectrum simulation. If the normalized characteristic lineshape that describes broadening due to the spectrometer, $\psi$, is known, then it may be convolved with the simulated spectrum to generate a prediction of the final measured transmission spectrum, $\eta_{m}$, as shown in Eq. (3).

$$
\begin{gathered}
\eta(\tilde{\nu})=\frac{I(\tilde{\nu})}{I_{0}(\tilde{\nu})}=\exp \left(-n X_{O H} l \sigma_{a b s}(T, P, \tilde{\nu})\right) \\
\eta_{m}(\tilde{\nu})=\int \eta(\theta-\tilde{\nu}) \psi(\theta) d \theta
\end{gathered}
$$

Once the measured spectrum is calculated, it must be resampled at the same spectral sampling frequency as the actual measured spectrum. In order to do this, a transformation from pixel number to wavenumber or wavelength is required. For small bandwidths, a linear function may be an adequate form of transformation for a dispersive spectrometer like the one used in this work. However, the spectral bandwidth of the measurements in this work was large enough that the dispersion varied significantly from one end of the measured spectrum to the other. A quadratic functional form for the transformation was found to adequately account for the higher order correction terms needed to correct for this. The three parameters describing that transformation function were floated in the spectral fitting algorithm described in section IV.A so that the optimum transformation for resampling of the simulated spectrum was used in each spectral fit.

\section{Experimental Methods}

\section{III.A. Spectrometer}

The spectrometer used in this work is a retrofitted McPherson 216.5 monochromator which has been configured to operate as a spectrograph and couple with a CCD detector. The process of reconfiguring the monochromator is similar to what was undertaken by Airey ${ }^{25}$ where the exit slit is removed and the active area of the imaging sensor is placed at the focal plane of the Czerny-Turner design. An Andor Newton 940 CCD camera with a BU-2 UV-enhanced sensor was chosen as the detector because of it's high responsivity below $300 \mathrm{~nm}(\mathrm{QE} \approx 60 \%)$ and relatively high pixel density (13.5 $\mu \mathrm{m}$ center-to-center pixel spacing $)$. In 
order to achieve proper placement of the sensor and avoid interference between the housing of the instrument and the camera, the camera is mounted upside down onto a support suspended from the top of the detector chamber of the instrument. The length of the support, and therefore the vertical position of the sensor, can be adjusted at installation via a threaded rod while a 2-dimensional rotating translation stage allow for free adjustment of the horizontal location and angle of the sensor. A custom back plate blocks stray light from entering the detector chamber and has a mounted fan to cool the camera. Light is coupled to the spectrograph via a fiber-optic cable which is located and maintained proximal to the entrance slit by a translation stage that is rigidly mounted onto the spectrograph housing.

The spectrograph uses a grating with a groove density of 2400 grooves $/ \mathrm{mm}$. The resulting bandwidth that can be captured by the detector is $19.4 \mathrm{~nm}$ with a spectral sampling spacing of $9.5 \mathrm{pm}$ between each pixel near $285 \mathrm{~nm}$. Because the uncertainty in the spectral fitting process increases with the level of noise in the measured transmission spectrum, it is important to achieve as high of a signal-to-noise ratio (SNR) as possible in the measured spectra. In pursuit of better SNRs, the measurements shown in this work are all averages over 1000 consecutively captured spectra. The acquisition rate of the detector was approximately 125 spectra/second and so the duration of each measurement was about 8 seconds.

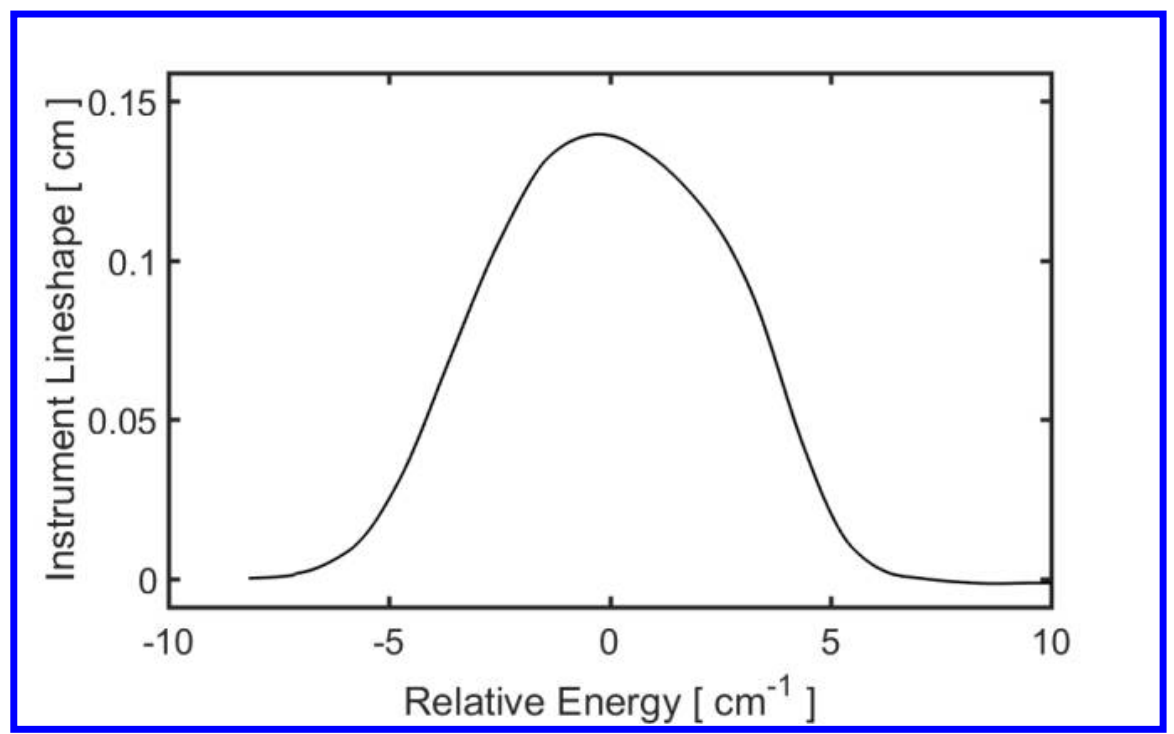

Figure 1 - Characteristic lineshape of the spectrometer as inferred from an average of effectively isolated absorption lines.

A critical part of inferring temperature and concentration values from measured spectra is accurate estimation of spectral broadening due to the spectrometer. All dispersive spectrometers cause some degree of spectral broadening due to their finite resolution. It is critical to have adequately characterized the spectral shape of the broadening in order to perform the type spectral derivative co-fitting described in section IV.A. Because the instrument lineshape is significantly broader than the absorption lines in the $\mathrm{OH}$ spectrum, an isolated absorption line can be approximated as a delta function compared to the instrument lineshape. This means that the shape of an isolated line as measured by the instrument approximates the characteristic instrument lineshape. Fortunately, there are four lines (specifically $\mathrm{Q}_{1}(12), \mathrm{Q}_{1}(13), \mathrm{Q}_{1}(14)$, and $\mathrm{P}_{1}(10)$ ) in the bandwidth that is captured in the measured spectra which are effectively isolated because adjacent lines exhibit orders of magnitude weaker absorption. Averaging the normalized lineshapes of these four lines from five different measured spectra gives the instrument lineshape estimate shown in figure 1. This lineshape is convolved with simulated spectra as described in Eq. (3) to account for the effect of instrument broadening.

\section{III.B. LEDs and Optics}

The excitation source used in this work was a UV LED with an emitted spectrum that is approximately a Gaussian distribution with a central wavelength of $285 \mathrm{~nm}$ and a spectral full-width at half maximum (FWHM) of $12 \mathrm{~nm}$. The maximum total optical power output of the LED is $0.8 \mathrm{~mW}$. The LED was chosen to probe the $v^{\prime}=1 \leftarrow v^{\prime \prime}=0$ vibrational band of the $\mathrm{A}^{2} \Sigma^{+} \leftarrow \mathrm{X}^{2} \Pi$ electronic transition.

In the experimental configuration used in this work, the LED is approximately collimated by a $35 \mathrm{~mm}$ 
focal length plano-convex lens. The roughly collimated beam is then focused by a $300 \mathrm{~mm}$ focal length lens onto a $1.5 \mathrm{~mm}$ pinhole in order to reduce the size cross-sectional width of the beam. A $75 \mathrm{~mm}$ focal length biconvex lens focuses the UV light into as thin of a beam as can be achieved along the absorption path length. A pair of plano-convex lenses re-collimate and focus the light onto the end of the fiber-optic cable.

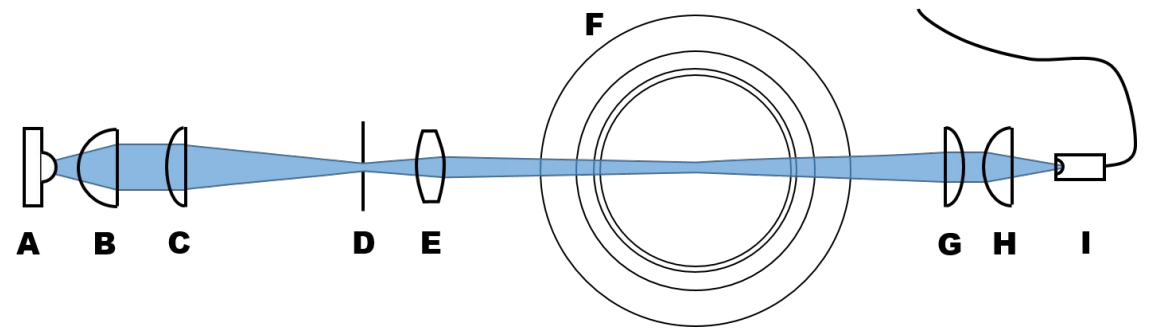

Figure 2 - Schematic diagram of the experimental optical configuration. A: UV LED, B: 35 $\mathrm{mm}$ collimating lens, C: $300 \mathrm{~mm}$ focusing lens, D: $1.5 \mathrm{~mm}$ pinhole, E: $75 \mathrm{~mm}$ biconvex lens, $\mathrm{F}$ : McKenna burner, G: $300 \mathrm{~mm}$ collimating lens, H: $50 \mathrm{~mm}$ focusing lens, I: Fiber-optic adapter.

The spectrally and spatially distributed nature of the LED light source as well as the aberrations introduced by the plano-convex lenses limit the degree of spatial resolution that can be achieved over the surface of the burner. Arranging the optical setup centers around a trade-off between the beam diameter and the strength of the measured transmission signal. Using a smaller pinhole, beam spot sizes with spatial FWHMs of less than $700 \mu \mathrm{m}$ at the edge of the burner and $125 \mu \mathrm{m}$ at the beam waist could be achieved. However, in this configuration a reduction of collected power of over $97 \%$ was observed. For this reason, the $1.5 \mathrm{~mm}$ pinhole was used. Measurements of the resulting beam's spatial profile are shown in figure 3 . These measured profiles suggest that the spatial FWHM of the beam was an average of approximately $1 \mathrm{~mm}$ along the absorption path length.

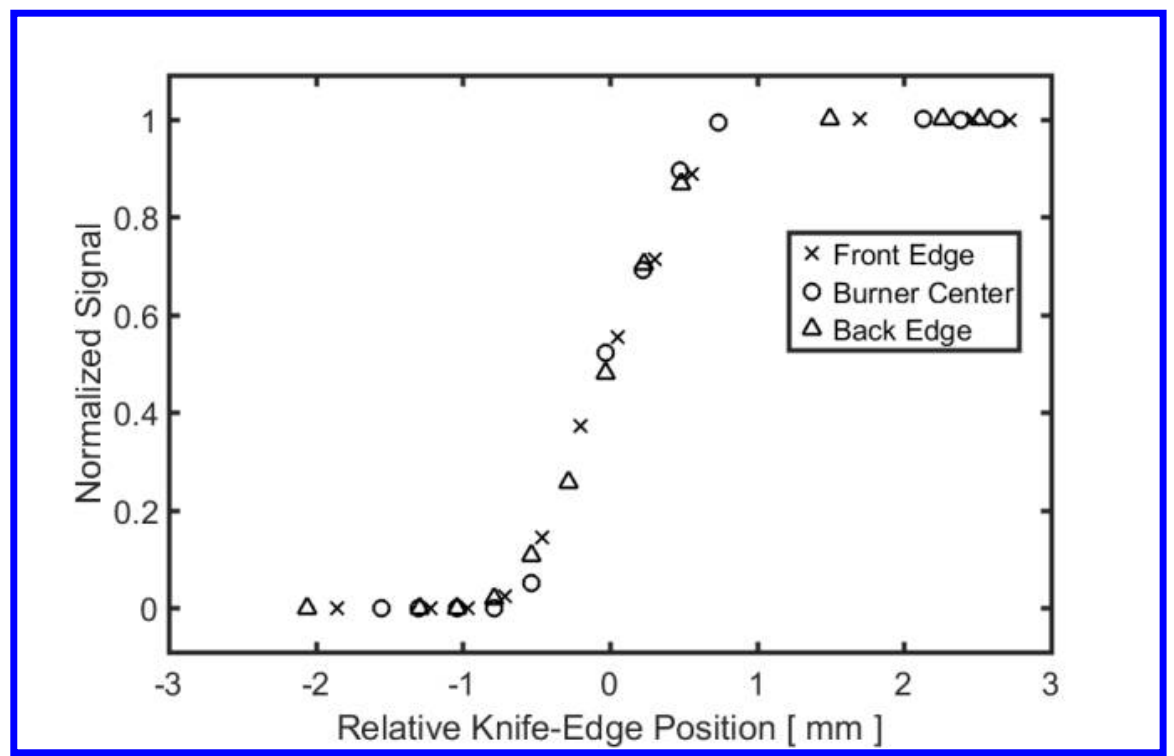

Figure 3 - Measurements of the distribution of optical power in the beam by traversing a knife-edge across the beam. Measurements of the beam profile are shown for locations along the absorption path at each edge of the burner and the center of the burner.

It is important to note that all UV LEDs that operate in this power regime exhibit some type of drift, due to thermal effects, in emitted optical power and spectral content. One strategy to mitigate this is to implement a thermal control system to maintain the temperature of the LED as consistently as possible. However, because the time-scale of these variations is on the order of minutes while each measured spectra can be captured in seconds, quite small changes in the LED output are observed over the course of a single measurement. For this reason, an alternative strategy has been adopted in this work where the emitted spectrum of the LED is measured immediately before and after each spectral measurement in a flame. The 
typical temporal separation between the before and after LED spectral measurements was about 2 minutes and, as expected, the average deviation between the two measured spectral was $\ll 0.1 \%$. An average of the before and after spectra was used as an estimate of $I_{0}(\lambda)$ for calculation of the transmission spectrum for each measurement point.

\section{III.C. Burner}

A porous-plug, flat-flame McKenna burner was used to generate laminar premixed methane-air flames as targets for measurements. The burner has a circular porous plug flow outlet with a diameter of $6 \mathrm{~cm}$ surrounded by a thin curtain of inert buffer flow ( $\mathrm{N}_{2}$ in this work). The burner is cooled by a water circuit providing room temperature $(295 \mathrm{~K})$ water at a flow rate of approximately 0.6 liters per minute. Three rotameters control the flow of methane, air, and nitrogen (for the buffer curtain) to the burner. Based on previous measurements of the radial flame temperature profile of the burner, it is estimated to have an effective absorption path length of $5.4 \mathrm{~cm}$ across the full diameter of a burner-stabilized flame.

\section{Results and Discussion}

\section{IV.A. Spectral Fitting}

In order to extract information from measured spectra, some form of spectral fitting must be implemented. Initial approaches to fitting flame measurements involved direct fitting of the absorbance spectrum using either non-linear least squares algorithms or grid searches using precomputed libraries of simulated absorbance spectra. These direct absorbance fitting techniques produced limited accuracy and precision for two primary reasons. The first being that the spectrometer used in these measurements exhibited broadening that is many times larger than the widths of the lines in the absorption spectrum that was measured. More importantly, the instrument broadening is larger than the spacing between spectral lines in most of the absorption spectrum. Because of this, most of the spectral features observed in measurements are combinations of several different lines, likely having different temperature dependencies, that have been broadened together. This requires an absorbance fitting method to be highly sensitive to subtle changes in magnitudes and shapes of the absorbance features, which is obstructed by the second reason that direct absorbance fitting is ineffective.

The second difficulty with direct absorbance spectrum fitting is the overwhelming influence of baseline error on the sum of squared fitting errors. Due to phenomena that cause spurious attenuation, such as beam steering through the flame and thermal drift in the LED output, smooth, low-amplitude baseline variations are observed in the measured absorbance spectrum. These small variations contain no relevant information about the conditions of interest, but because they are smooth (low frequency) they exhibit a large influence on the sum of squared errors of the absorbance spectrum fit. As a consequence, fitting algorithms will be much more sensitive to variations in the baseline error than variations in real absorbance features. This will cause the algorithms to manipulate the $\mathrm{OH}$ mole fraction to best reduce errors due to baseline variation while matching spectral features effectively become an afterthought, leading to large measurement uncertainties.

These issues can be mitigated using a technique commonly used in FTIR community known as resolution enhancement by derivative fitting. ${ }^{22}$ In this approach, the second derivative, what we will call the curvature (note that this is only an approximation of the true mathematical quantity named curvature), of the measured spectrum is estimated from spectral measurements and is fit using curvatures estimated in the same way from simulated spectra. The curvature of the absorbance spectrum is much more sensitive to variations in the latent spectral lines that have been broadened together than is the magnitude of the absorbance spectrum itself. In this way, information that is obfuscated by broadening can be, to a significant degree, elucidated by curvature fitting, which is reason for the term "resolution enhancement". The influence of the baseline error is also virtually eliminated because the curvature of the low-frequency, low-amplitude baseline variation is far lower in magnitude than that of the real spectral features. This leaves random noise in the absorbance signal and any possible unknown biases that might be introduced by the modeling as the two most significant sources of uncertainty in the results of the spectral curvature fitting.

In particular, this work uses a co-fitting approach proposed and analyzed by Baldassarre et. al. ${ }^{23}$ This technique fits both the absorbance spectrum and its curvature using a least-squares algorithm that weights the absorbance and curvature errors relative to each other. Through trial and error, a weighting value which makes the magnitude of the squared error in the fit of the curvature approximately 25 times that of squared error associated with the absorbance spectrum fit was chosen. The fitting parameters which are floated in 


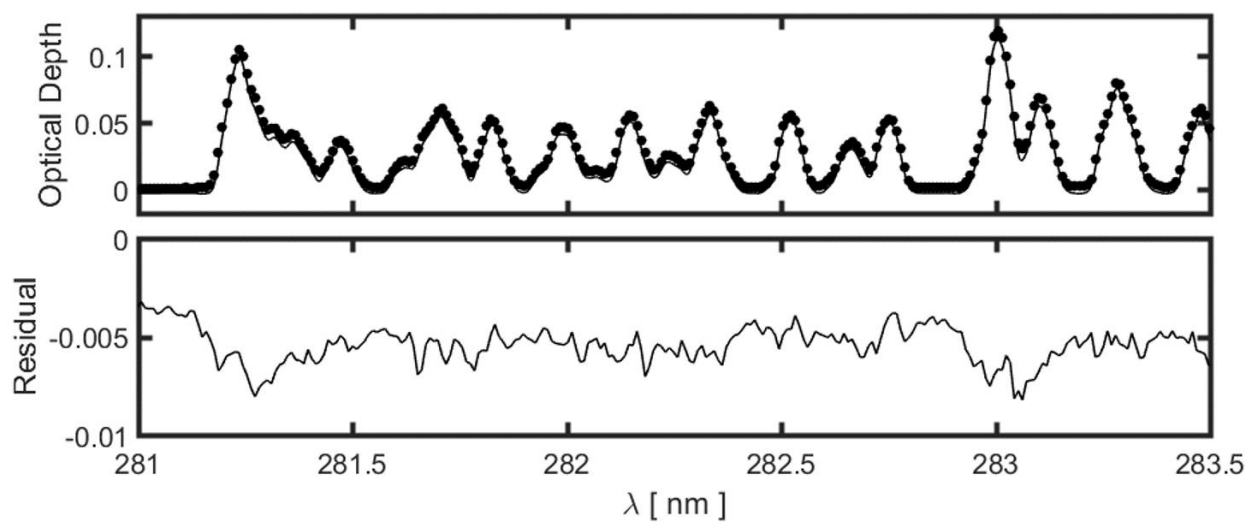

Figure 4 - The absorbance spectrum fit from an optimized spectral co-fitting of a methane-air flame measurement. The solid line in the upper plot is the best fit simulated spectrum and the dots are experimentally measured points.

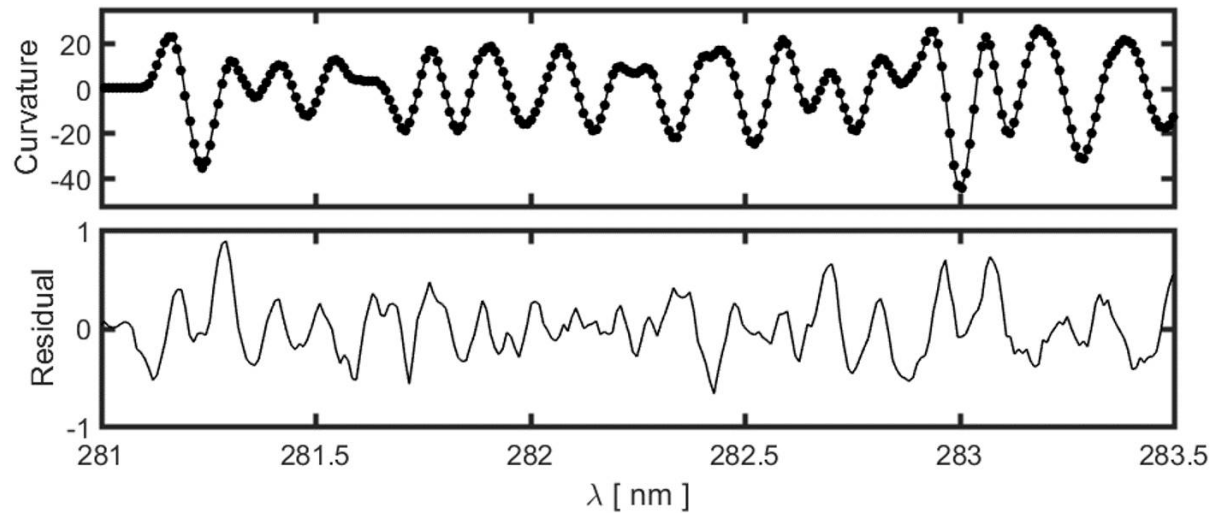

Figure 5 - The fit of the second derivative (curvature) of an absorbance spectrum from the same optimized spectral co-fitting that is shown in figure 4. The solid line represents the curvature of the simulated spectrum and the dots indicate measured points.

the least-squares algorithm are the temperature and $\mathrm{OH}$ mole fraction inputs to the $\mathrm{OH}$ absorption model and 3 parameters defining a quadratic transform between pixel number and wavelength. The LevenbergMarquardt least-squares algorithm was used to optimize the spectral co-fitting of the measured absorbance spectra and their curvatures. The second derivative of the measured and simulated spectra were estimated using a third-order Savitsky-Golay convolution with a window size of 15 points. ${ }^{26-28}$

An example of a co-fitting of an absorbance spectrum and its second derivative is shown in figure 4 and figure 5. The absorbance spectrum is displayed in units of optical depth consistent with how the spectral fits are computed, but this can be directly converted to absorbance by dividing by a factor of the natural logarithm of 10 . Only a portion of the spectrum that was fit is shown for clarity and the results of the fit gave optimum values for temperature and $\mathrm{OH}$ mole fraction of $\mathrm{T}=1885 \mathrm{~K}$ and $\mathrm{X}_{O H}=1899 \mathrm{ppm}$. Note the nearly constant component of error in the absorbance spectrum fit shown in figure 4 . This is an example of the baseline errors which limit the effectiveness of solely fitting the absorbance spectrum. As is clear in figure 5, this baseline error is not present in the second derivative of the spectrum and so by weighting the curvature fit heavily, the sensitivity to the baseline error can be mitigated.

\section{IV.B. Flame Measurements}

Measurements in methane-air flames have been performed as an initial test of the broadband absorption diagnostic. In particular, a stoichiometric flame stabilized on the McKenna burner using the flow rates 
summarized in table 1 was examined. Measurements were taken at increasing distances above the surface of the burner to allow for inferred measurements of the variation in the $\mathrm{OH}$ rotational temperature and concentration along the axial dimension of the flame. The temperature and concentration values were inferred by applying the previously described spectral co-fitting approach to a section of the spectrum from $279.5 \mathrm{~nm}$ to $287.1 \mathrm{~nm}$ which captures as much of the $\left(v^{\prime}=1, v "=0\right)$ band as possible while avoiding any contributions from the $\left(v^{\prime}=2, v^{\prime \prime}=1\right)$ band for simplicity of modeling.

Table 1 - Conditions used in flame measurements.

\begin{tabular}{lrl} 
Property & Value & Units \\
\hline Equivalence Ratio & 1.0 & \\
Pressure & 1.0 & atm \\
$\mathrm{CH}_{4}$ Flow Rate & 2.13 & slpm \\
Air Flow Rate & 20.24 & slpm \\
Cooling Water Flow Rate & 0.6 & $\mathrm{lpm}$
\end{tabular}

Figures 6 and 7 show the inferred temperature and $\mathrm{OH}$ mole fraction measurements from the stoichiometric methane-air flame. For comparison, a Chemkin simulation of a burner-stabilized stoichiometric methane-air flame calculated using the GRI-Mech 3.0 chemistry mechanism is shown as the solid black lines. However, the Chemkin model was run as a purely adiabatic system while we know that there were significant but unquantified amounts of heat loss in the flame used in the experimental measurements. Because the thermal boundary conditions are necessarily incorrect and radiative heat loss throughout the flame is neglected, there is not expected to be direct agreement between the calculation and the measurements. The simulation results are included to give context to the trends and magnitudes of the flame measurements.

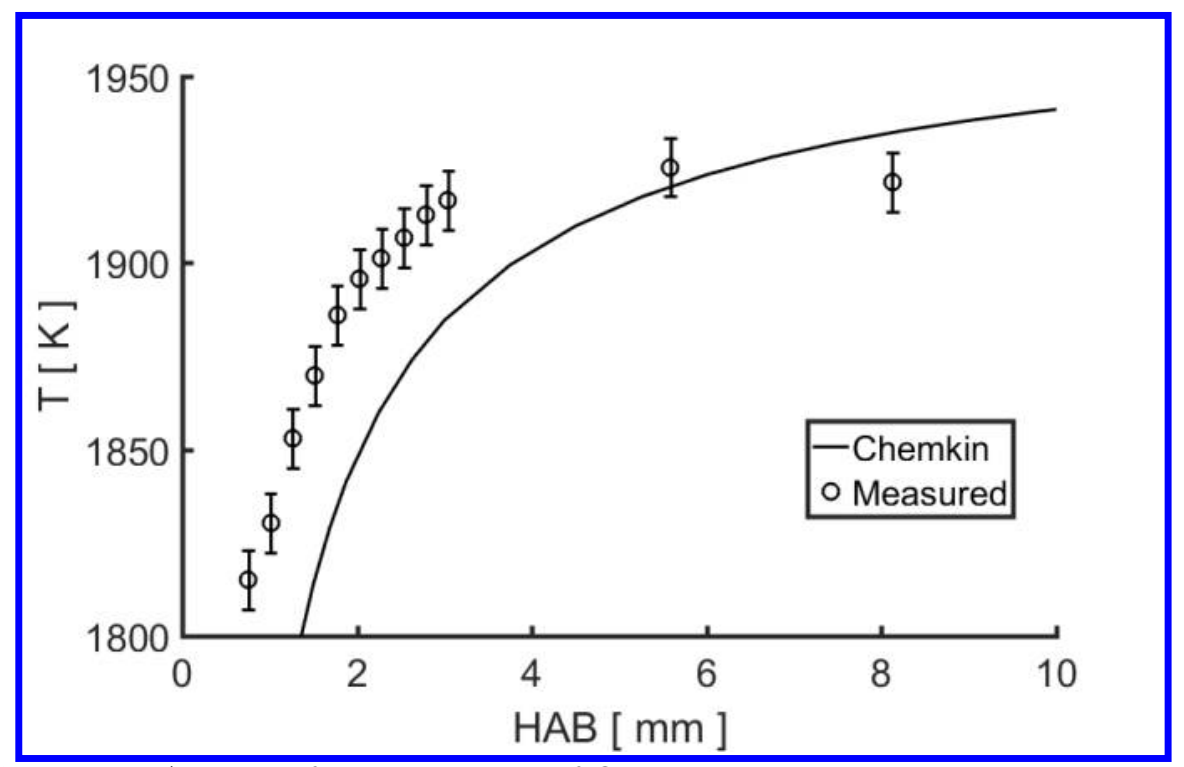

Figure 6 - A series of measurements of $\mathrm{OH}$ rotational temperature variation in a stoichiometric methane-air flame with height above the burner surface. The solid line shows a calculation performed in Chemkin of an adiabatic burner-stabilized flame for comparison.

Error bars represent a $95 \%$ confidence interval.

One phenomenon that was present in these measurements, the consequences of which may be seen through careful inspection of the region closest to the burner in figure 6, was a spatial averaging across the width of the beam along the axial direction. The effect of this is to weight the contributions to the observed spectrum by the horizontal width of the beam and the $\mathrm{OH}$ concentration at each axial position in the beam cross-section. Because the beam width is on the order of the length scale of the gradients expected in this flame near the surface of the burner $(\approx 1 \mathrm{~mm})$, this is where the spatial averaging can be seen to have its greatest effect. In figure 6 , the two measurements that were made closest to the burner surface experience 


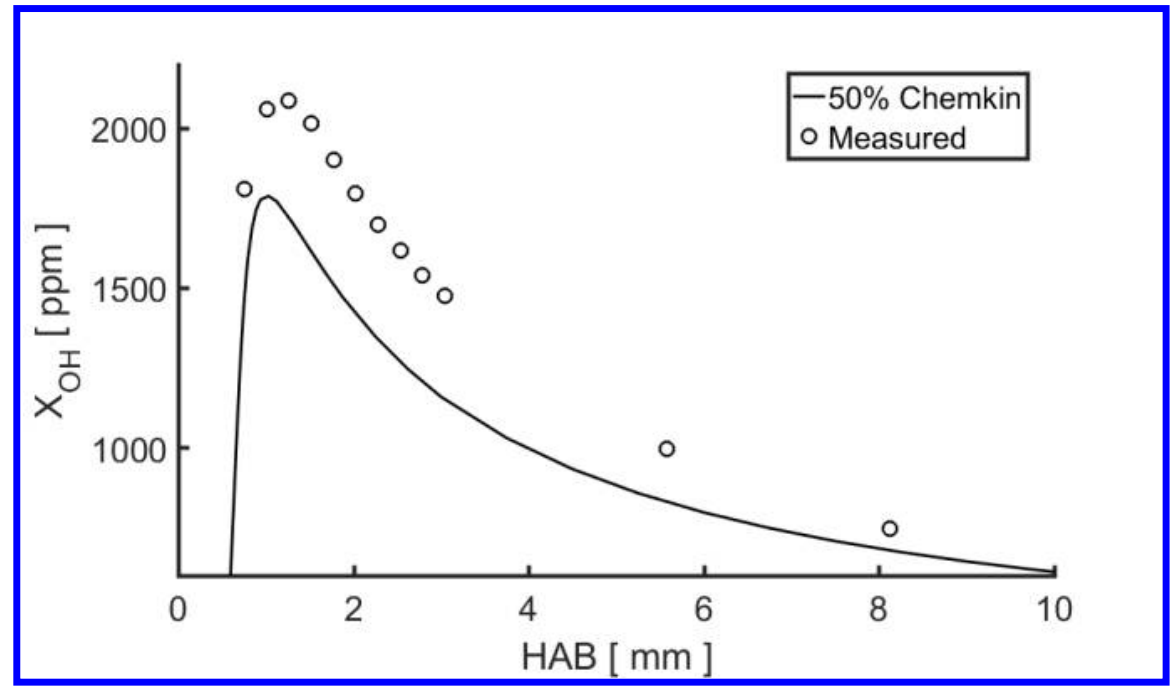

Figure 7 - A series of measurements of $\mathrm{OH}$ species concentration variation in a stoichiometric methane-air flame with height above the burner surface. The solid line shows a calculation performed in Chemkin of an adiabatic burner-stabilized flame with the magnitude reduced by $50 \%$ for ease of comparison.

a much greater influence of the axial locations in the top of the beam with high temperatures and high $\mathrm{OH}$ concentrations than of the upstream locations with lower temperature and lower $\mathrm{OH}$ concentrations. While a more sophisticated analysis will be required to quantify the effect of the spatial averaging, it is reasonable to expect that this will cause the temperature measurements in the region closer to the surface of the burner to be inflated. This would yield lower measured spatial temperature gradients near the surface of the burner than would be seen with perfect spatial resolution. This is consistent with the results in this work.

Despite the distortions due to spatial averaging, the measurements generally follow a trend in both temperature and $\mathrm{OH}$ mole fraction that would be expected in this type of flame. It should also be noted that the $\mathrm{OH}$ mole fractions computed by Chemkin have been reduced by a factor of 2 in figure 7 . Influences of thermal effects that were neglected in the flame simulations likely cause a large component of the discrepancy and so the results were displayed with a scaling factor to ease the reader's qualitative comparison of the profiles. Future efforts to improve the quantification and simulation of heat losses, spatial resolution of the measurements, and fine-tuning of a target flame with lower spatial gradients are expected to enable a more rigorous verification analysis of measurements.

\section{IV.C. Estimation and Interpretation of Uncertainty}

Uncertainty in the flame measurements shown in this work was estimated using an empirical approach. A series of relatively low SNR single-shot spectral measurements was captured and then analyzed at varying noise levels by averaging together different numbers of single-shot spectra. The statistical spread of a number of best fit values using these averaged spectra was used to estimate the uncertainty for temperature and mole fraction measurements inferred from spectral fitting. Spectra with twice the noise level of the measurements shown in figures 6 and 7 were used to ensure that the estimate of uncertainty would be a conservative one. The standard deviations associated with uncertainty due to random errors were estimated to be $4.04 \mathrm{~K}$ for temperature and $6.4 \mathrm{ppm}$ for $\mathrm{OH}$ mole fraction.

It is important to note that, because of the nature of inferring values through spectral fitting, errors in the temperature and $\mathrm{OH}$ mole fraction measurements are not independent. In fact, the inferred values of temperature and $\mathrm{OH}$ concentration are strongly correlated with each other. Sets of inferred temperature and $\mathrm{OH}$ concentrations in the empirical uncertainty analysis typically had a correlation coefficient of approximately 0.95 between the two quantities. For this reason, uncertainties in each of the values should not be considered independently, but should always be viewed in the context of their high correlation. To demonstrate this concept, it is best to view each of these measurements in the temperature and $\mathrm{OH}$ concentration phase space as shown in figure 8 . The solid curve in the figure is a cubic spline fit connecting measurements in the flame 


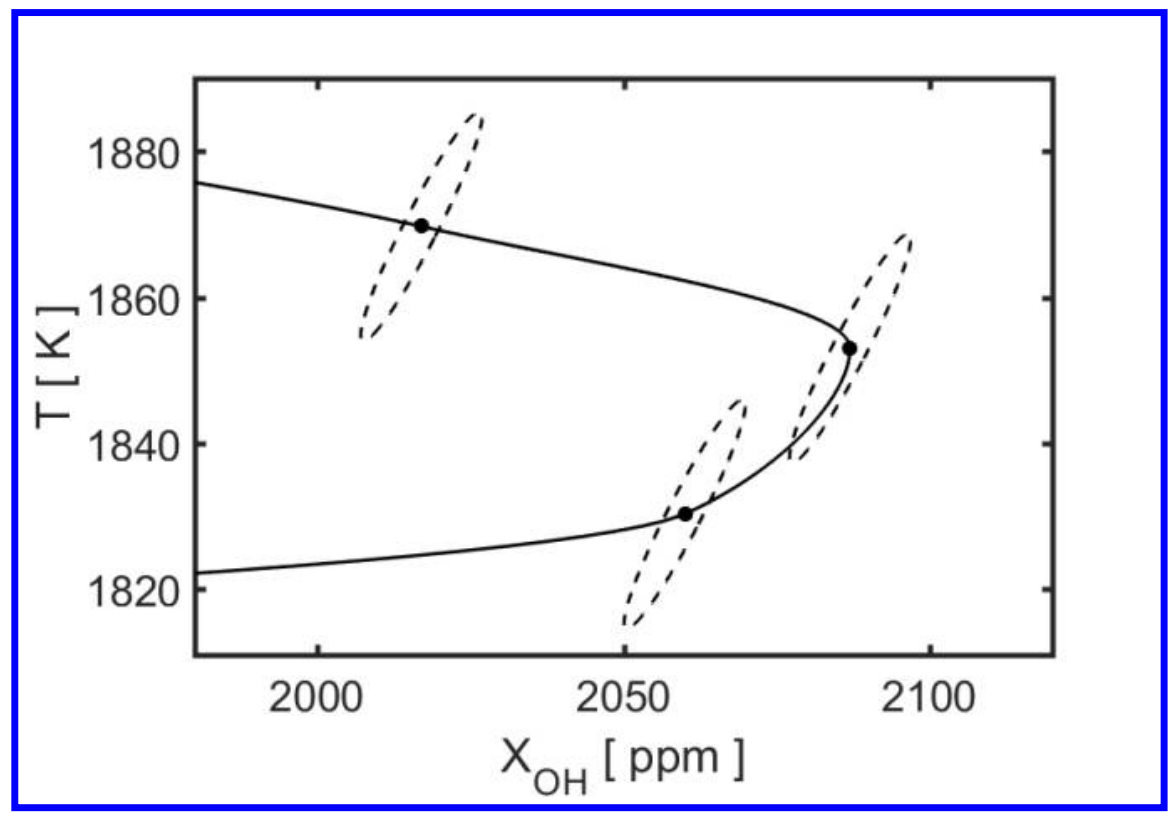

Figure $\mathbf{8}$ - A demonstration of uncertainty ellipses associated with correlated measurements of temperature and $\mathrm{OH}$ concentration from LED absorption analysis. These are shown in a temperature and $\mathrm{OH}$ mole fraction phase space. The solid line approximates the locus of all axial locations along the flame. Three dots indicate individual measurement points and the ellipses show by the dashed lines enclose the $95 \%$ confidence regions of the measured values.

as an approximation of the locus of all axial locations in the flame. In this view, confidence regions will not be squares or even circles in phase space, but elliptic regions due to the nonzero correlation between the variables. Examples of these elliptic confidence regions are shown in figure 8 by the dashed lines enclosing the $95 \%$ confidence regions ${ }^{29}$ of the measured points indicated by the dots.

\section{Conclusion}

A novel broadband absorption diagnostic for quantitative measurements of combustion radical species concentration and rotational temperature has been proposed. This work outlines a preliminary approach to the experimental and theoretical methods that could be used to carry out such measurements. Chief among the insights discovered in the course of analyzing these measurements is the effectiveness of derivative fitting for inferring temperature measurements, specifically the use of spectral absorbance and spectral curvature co-fitting. Measurements of temperature and $\mathrm{OH}$ species concentration were made in a laminar, premixed stoichiometric methane-air flame to demonstrate the use and capabilities of the diagnostic.

Conservative estimates of the magnitude of uncertainties associated with these types of measurements were made. It has been shown that an awareness of the high correlation between inferred temperature and $\mathrm{OH}$ concentration is necessary in order to achieve an understanding of the uncertainty in measurements made using this diagnostic. This is clearly seen when data are viewed in the temperature and $\mathrm{OH}$ concentration phase space where confidence regions take on concentric elliptic shapes.

Future work on the development of this diagnostic will include improvement of the verification measurements such that a quantitative evaluation of the capabilities of the diagnostic may be made. A full analysis of the effects of spatial averaging, choice of spectral fitting parameters, and of measurement uncertainty will be undertaken. Applications of the diagnostic which offer the opportunity to leverage its unique ability to measure the population fractions of many energy levels simultaneously will be pursued.

\section{Acknowledgments}

The work presented here is partially supported by the Air Force Office of Scientific Research under Grant No. FA9550- 14-1-0396 with Dr. Chiping Li as Technical Monitor. Logan White is supported by the 
Department of Defense (DoD) through the National Defense Science \& Engineering Graduate Fellowship (NDSEG) Program. The authors would like to acknowledge and thank Justin Spott and Andor Technology for providing Andor Newton 970 and 940 series CCD cameras that were used to make the initial measurements for this project.

\section{References}

${ }^{1}$ Dally, B., Masri, A., Barlow, R., and Fiechtner, G., "Instantaneous and Mean Compositional Structure of Bluff-Body Stabilized Nonpremixed Flames," Combustion and Flame, Vol. 114, No. 1-2, 1998, pp. 119-148.

${ }^{2}$ Kelman, J. B. and Masri, A. R., "Quantitative technique for imaging mixture fraction, temperature, and the hydroxyl radical in turbulent diffusion flames," Applied Optics, Vol. 36, 1997, pp. 3506-3514.

${ }^{3}$ Cattolica, R., "OH rotational temperature from two-line laser-excited fluorescence." Applied Optics, Vol. 20, No. 7, 1981, pp. 1156-1166.

${ }^{4}$ Crosley, D. R. and Smith, G. P., "Rotational Energy Transfer and LIF Temperature Measurements," Combustion and Flame, Vol. 44, 1982, pp. 27-34.

${ }^{5}$ Kelman, J. B. and Masri, A. R., "Quantitative imaging of temperature and $\mathrm{OH}$ in turbulent diffusion flames by using a single laser source." Applied Optics, Vol. 33, No. 18, 1994, pp. 3992-9.

${ }^{6}$ Seitzman, J. M., Hanson, R. K., Debarber, P. A., and Hess, C. F., "Application of quantitative two-line OH planar laser-induced fluorescence for temporally resolved planar thermometry in reacting flows." Applied Optics, Vol. 33, No. 18, 1994, pp. 4000-4012.

${ }^{7}$ Allen, M. G., McManus, K. R., Sonnenfroh, D. M., and Paul, P. H., "Planar laser-induced-fluorescence imaging measurements of $\mathrm{OH}$ and hydrocarbon fuel fragments in high-pressure spray-flame combustion." Applied Optics, Vol. 34, No. 27, 1995, pp. $6287-300$.

${ }^{8}$ Arnold, A., Bombach, R., Käppeli, B., and Schlegel, A., "Quantitative measurements of OH concentration fields by two-dimensional laser-induced fluorescence," Applied Physics B: Lasers and Optics, Vol. 64, No. 5, 1997, pp. 579-583.

$>{ }^{9}$ Atakan, B., Heinze, J., and Meier, U., "OH laser-induced fluorescence at high pressures: spectroscopic and twodimensional measurements exciting the A-X $(1,0)$ transition," Applied Physics B: Lasers and Optics, Vol. 64, No. 5, 1997, pp. 585-591.

${ }^{10}$ Kohse-Höinghaus, K., Meier, U., and Attal-Trétout, B., "Laser-induced fluorescence study of OH in flat flames of 1-10 bar compared with resonance CARS experiments." Applied Optics, Vol. 29, No. 10, 1990, pp. 1560-9.

${ }^{11}$ Seitzman, J. M. and Hanson, R. K., "Comparison of excitation techniques for quantitative fluorescence imaging of reacting flows," AIAA Journal, Vol. 31, No. 3, 1993, pp. 513-519.

${ }^{12}$ Battles, B. E. and Hanson, R. K., "Laser-induced fluorescence measurements of $\mathrm{NO}$ and $\mathrm{OH}$ mole fraction in fuellean, high-pressure (110 atm) methane flames: Fluorescence modeling and experimental validation," Journal of Quantitative Spectroscopy and Radiative Transfer, Vol. 54, No. 3, 1995, pp. 521-537.

$\checkmark{ }^{13}$ Bruggeman, P., Cunge, G., and Sadeghi, N., "Absolute OH density measurements by broadband UV absorption in diffuse atmospheric-pressure $\mathrm{HeH}_{2} \mathrm{O}$ RF glow discharges," Plasma Sources Science and Technology, Vol. 21, No. 3, 2012 , pp. 035019.

$\checkmark{ }^{14}$ Dilecce, G., Ambrico, P. F., Simek, M., and Benedictis, S. D., "OH density measurement by time-resolved broad band absorption spectroscopy in an ArH 2 O dielectric barrier discharge," Journal of Physics D: Applied Physics, Vol. 45, No. 12, 2012, pp. 125203.

${ }^{15}$ Verreycken, T., Mensink, R., Horst, R. V. D., Sadeghi, N., and Bruggeman, P. J., "Absolute OH density measurements in the effluent of a cold atmospheric-pressure ArH2O RF plasma jet in air," Plasma Sources Science and Technology, Vol. 22, 2013 , pp. 1.

16Xiong, Q., Yang, Z., and Bruggeman, P. J., "Absolute OH density measurements in an atmospheric pressure dc glow discharge in air with water electrode by broadband UV absorption spectroscopy," Journal of Physics D: Applied Physics, Vol. 48, No. 42, 2015, pp. 424008.

$\checkmark 17$ Thompson, J. E. and Myers, K., "Cavity ring-down lossmeter using a pulsed light emitting diode source and photon counting," Measurement Science and Technology, Vol. 18, No. 1, 2006, pp. 147-154.

${ }^{18}$ Degner, M., Damaschke, N., Ewald, H., and Lewis, E., "High resolution led-spectroscopy for sensor application in harsh environment," Instrumentation and Measurement Technology Conference (I2MTC), 2010 IEEE, 2010, pp. 1382-1386.

$\checkmark{ }^{19}$ Degner, M., Ewald, H., and Lewis, E., "Novel sensor cell design and algorithm to online realize stable and cost effective optical concentration measurements at fluctuating light source situations," 2011 Fifth International Conference on Sensing Technology, 2011, pp. 1950-1953.

${ }^{20}$ Degner, M. and Ewald, H., "LED-spectroscopy based on multi quantum well emitter," 2012 International Conference on Electromagnetics in Advanced Applications, 2012, pp. 840-843.

${ }^{21}$ Schorsch, S., Kiefer, J., Leipertz, A., Li, Z., and Aldén, M., "Detection of Flame Radicals Using Light-Emitting Diodes," Vol. 64, No. 12, 2010, pp. 1330-1334.

${ }^{22}$ Mark, H., Chemometrics in spectroscopy, Elsevier/Academic Press, Amsterdam Boston, 2007.

${ }^{23}$ Baldassarre, M., Li, C., Eremina, N., Goormaghtigh, E., and Barth, A., "Simultaneous Fitting of Absorption Spectra and Their Second Derivatives for an Improved Analysis of Protein Infrared Spectra," Molecules, Vol. 20, No. 7, jul 2015, pp. 12599-12622.

${ }^{24}$ Luque, J. and Crosley, D., "LIFBASE: Database and Spectral Simulation," SRI International Report MP, Vol. 99-009, 1999.

${ }^{25}$ Airey, D., "Performance of a fibre-optic coupled, high-resolution spectrograph with CCD camera recording of solar H $\alpha$ and H $\beta$ absorption spectra," Journal of the British Astronomical Association, Vol. 116, No. 6, 2006, pp. 309-319. 
${ }^{26}$ Savitzky, A. and Golay, M. J. E., "Smoothing and Differentiation of Data by Simplified Least Squares Procedures." Analytical Chemistry, Vol. 36, No. 8, jul 1964, pp. 1627-1639.

${ }^{27}$ Steinier, J., Termonia, Y., and Deltour, J., "Smoothing and differentiation of data by simplified least square procedure," Analytical Chemistry, Vol. 44, No. 11, sep 1972, pp. 1906-1909.

${ }^{28}$ Madden, H. H., "Comments on the Savitzky-Golay convolution method for least-squares-fit smoothing and differentiation of digital data," Analytical Chemistry, Vol. 50, No. 9, aug 1978, pp. 1383-1386.

${ }^{29}$ Hoover, W. E., "Algorithms For Confidence Circles and Ellipses," NOAA Technical Report NOS 107 C\&GS 3, NOAA Charting and Geodetic Services, Rockville, MD, sep 1984. 